\title{
Lattice Supersymmetry: Some Ideas from Low Dimensional Models
}

\author{
Alessandro D’Adda ${ }^{* a}$, Alessandra Feo $^{a}$, Issaku Kanamori ${ }^{a}$, Noboru Kawamoto $^{b}$ and \\ Jun Saito ${ }^{b}$ \\ a Dipartimento di Fisica Teorica, Universita' di Torino and INFN Sezione di Torino \\ I-10125 Torino, Italy. \\ ${ }^{b}$ Department of Physics, Hokkaido University, Sapporo, 060-0810 Japan \\ E-mail: dadda@to.infn.it, feo@to.infn.it, kanamori@to.infn.it, \\ kawamoto@particle.sci.hokudai.ac.jp, \\ saito@particle.sci.hokudai.ac.jp
}

In the framework of the so called link approach we study exact lattice supersymmetry for the simplest supersymmetric model: $N=1$ supersymmetry in $D=1$. The model is described by a lattice with spacing $\frac{a}{2}$, thus containing twice as many sites as the conventional one. The boson and fermion are related through a $2 \pi / a$ momentum shift, which can provide an interpretation of them being species doublers to each other. An exactly supersymmetric lattice action can be written within this scheme in momentum representation, which however turns out to be non local in coordinate space.

The XXVII International Symposium on Lattice Field Theory - LAT2009

July 26-31 2009

Peking University, Beijing, China

\footnotetext{
*Speaker.
} 


\section{Introduction}

In recent years a number of lattice models with at least one exact supersymmetry at non-zero lattice spacing have been proposed ${ }^{1}$. Topological twist and orbifold projection are the main new ideas behind these approaches, which however allow in general only one exact lattice supersymmetry. The link approach was proposed to overcome this limitation and realize exactly on the lattice all supersymmetries in some models with extended supersymmetry $[2,3,4,5]$. The key new ingredient of this approach with respect to the ones mentioned above (to which it is however deeply related [6]) is the introduction of extended lattices with additional "fermionic" links on which supersymmetry charges sit. As a consequence of the link nature of the (super)symmetry charges, modified Leibnitz rules have to be applied when (super)charges act on a product of (super)fields. Hence both the consistency and the relevance of the link approach to the description of exact lattice supersymmetry has been questioned [7,8]. However it has been shown recently by some of the present authors that a consistent mathematical set up for the link approach can be given in terms of Hopf algebras [9]. The introduction of new links and new sites implies that a larger number of degrees of freedom is present in the link approach formulation, namely that the theory contains doublers both for bosons and fermions unless some mechanism is found to get rid of them. This is one motivation for looking at the simplest supersymmetric system: an $N=1$ supersymmetry in one space-time dimension. In spite of its simplicity the investigation of this model in the framework of the link approach offers some insight into some relevant problems, including the doublers mentioned above, and it is worth pursuing. This will be the subject of the present talk.

\section{2. $\mathrm{D}=1, \mathrm{~N}=1$ model}

The simplest supersymmetric model is a one dimensional model with just one supersymmetric charge. It is described in terms of a superfield:

$$
\Phi(x, \theta)=\varphi(x)+i \theta \psi(x)
$$

with a supersymmetry charge given by:

$$
Q=\frac{\partial}{\partial \theta}+i \theta \frac{\partial}{\partial x}
$$

and

$$
Q^{2}=i \frac{\partial}{\partial x}
$$

The free action is given by

$$
S=\frac{i}{2} \int d x d \theta D \Phi \frac{\partial \Phi}{\partial x}
$$

where $D$ is the super derivative. Because of the fermionic nature of the superspace integration volume no potential can be written in terms of the superfield and the theory is essentially free.

On the lattice the derivative $\frac{\partial}{\partial x}$ is replaced by finite shift $\Delta$, defined by:

$$
\Delta \Phi(x)=\Phi(x+a) \Delta,
$$

\footnotetext{
${ }^{1}$ For a review see [1] and references therein.
} 
where $x$ and $x+a$ are two neighboring sites separated by the lattice spacing $a$.

In the link approach a shift $a_{Q}$ is associated to the supersymmetry charge $Q$, and since eq. (2.3) is replaced by

$$
Q^{2}=i \Delta
$$

we have $a_{Q}=\frac{a}{2}$. The supersymmetric extended lattice is then given by integer multiples of $\frac{a}{2}$, thus doubling the number of the the original lattice sites. We assume here that $Q$ acts on the fields as a $\operatorname{shift}^{2}$ of $\frac{a}{2}$, just as $\Delta$ acts as a shift of $a$. The question now is: What makes this lattice different from just an ordinary lattice with spacing $\frac{a}{2}$ ? The answer is in the different behaviour of bosonic and fermionic fields with respect to shifts of $\frac{a}{2}$, namely with respect to supersymmetry transformations. In the continuum a constant bosonic field commutes with $Q$, hence for a constant field we have on the lattice:

$$
\varphi\left(x+\frac{a}{2}\right)-\varphi(x)=0
$$

which implies that $\varphi$ is constant on the lattice. A constant fermionic field instead anticommutes with $Q:\{Q, \psi\}=0$. If we assume that on the lattice $Q$ simply acts as a shift of $\frac{a}{2}$, then $\{Q, \psi\}=0$ implies for a constant fermionic field $\psi_{l}(x)$ :

$$
\psi_{l}\left(x+\frac{a}{2}\right)+\psi_{l}(x)=0
$$

namely

$$
\psi_{l}(x)=(-1)^{\frac{2 x}{a}} \psi_{0}
$$

where $\psi_{0}$ is a constant and $\frac{2 x}{a}$ is an integer on the lattice.

Physical fields are fluctuations around constant configurations. One can then tentatively write a superfield on the lattice as

$$
\Phi(x)=\varphi(x)+\frac{a^{\frac{1}{2}}}{2}(-1)^{\frac{2 x}{a}} \psi(x)
$$

where $\varphi(x)$ and $\psi(x)$ are smooth fields, in the sense that for instance $\psi\left(x+\frac{a}{2}\right)-\psi(x)$ is of order $a$ in the continuum limit. The smooth field $\psi(x)$ is related to the original lattice fermion $\psi_{l}(x)$ by the relation

$$
\psi_{l}(x)=(-1)^{\frac{2 x}{a}} \psi(x)
$$

so that $\psi_{l}(x)$ satisfies the smoothness condition $\psi_{l}\left(x+\frac{a}{2}\right)+\psi_{l}(x)=O(a)$. Eq. (2.10) resembles the usual superfield expansion with the sign factor $(-1)^{2 x / a}$ playing the role of $\theta$.

The supersymmetry transformations are given in the continuum by:

$$
\delta \Phi(x, \theta)=\alpha[Q, \Phi]
$$

The lattice equivalent is

$$
\delta \Phi(x)=a^{-\frac{1}{2}} \alpha(-1)^{\frac{2 x}{a}}\left(\Phi\left(x+\frac{a}{2}\right)-\Phi(x)\right),
$$

\footnotetext{
${ }^{2}$ This is in agreement with the matrix representation of lattice superspace given in [5]. Notice in particular that in this way $Q$ does not contain any Grassmann odd parameter.
} 
where the constant fermionic parameter $\alpha$ in the continuum has been consistently replaced on the lattice by $\alpha(-1)^{\frac{2 x}{a}}$. From here one can read the supersymmetric transformations for the component fields as

$$
\begin{aligned}
& \delta \varphi(x)=-\frac{\alpha}{2}\left[\psi\left(x+\frac{a}{2}\right)+\psi(x)\right] \underset{a \rightarrow 0}{\longrightarrow}-\alpha \psi(x), \\
& \delta \psi(x)=2 a^{-1} \alpha\left[\varphi\left(x+\frac{a}{2}\right)-\varphi(x)\right] \underset{a \rightarrow 0}{\longrightarrow} \alpha \frac{\partial \varphi(x)}{\partial x} .
\end{aligned}
$$

The supersymmetry transformations (2.14) and (2.15) have the correct structure, but they are still not the right ones. In fact the variation of $\varphi(x)$ at the 1.h.s. of (2.14) is not real: an $i$ factor is missing. In order to restore the hermiticity of the supersymmetry transformations symmetric finite differences must be used, introducing a shift of $\frac{a}{4}$ of the fermionic fields sites with respect to the bosonic ones. Hence, instead of writing the superfield on the lattice as in (2.10) we shall introduce $\Phi(x)$, with $x=n \frac{a}{4}$, defined by:

$$
\Phi(x)=\left\{\begin{array}{cl}
\varphi(x) & \text { for } x=n a / 2 \\
\frac{1}{2} a^{1 / 2} e^{\frac{2 i \pi x}{a}} \psi(x) & \text { for } x=(2 n+1) a / 4
\end{array}\right.
$$

Again the supersymmetry transformations can be written in terms of $\Phi(x)$ :

$$
\delta \Phi(x)=\alpha a^{-1 / 2} e^{\frac{2 i \pi x}{a}}[\Phi(x+a / 4)-\Phi(x-a / 4)] .
$$

By separating $\Phi(x)$ into its component fields according to (2.16) we find:

$$
\begin{aligned}
& \delta \varphi(x)=\frac{i \alpha}{2}\left[\psi\left(x+\frac{a}{4}\right)+\psi\left(x-\frac{a}{4}\right)\right] \underset{a \rightarrow 0}{\longrightarrow} i \alpha \psi(x), \\
& \delta \psi(x)=2 a^{-1} \alpha\left[\varphi\left(x+\frac{a}{4}\right)-\varphi\left(x-\frac{a}{4}\right)\right] \underset{a \rightarrow 0}{\longrightarrow} \alpha \frac{\partial \varphi(x)}{\partial x},
\end{aligned}
$$

where $x$ is an even multiple of $a / 4$ in (2.18) and an odd one in (2.19). As in the continuum case the commutator of two SUSY transformation is a translation, namely, on the lattice, a finite difference of spacing $a$. For instance we have for $\varphi(x)$ (the same applies to $\psi(x)$ ):

$$
\delta_{\beta} \delta_{\alpha} \varphi(x)-\delta_{\alpha} \delta_{\beta} \varphi(x)=2 i \alpha \beta[\varphi(x+a / 2)-\varphi(x-a / 2)] .
$$

To summarize: even in this extremely simple case exact supersymmetry on the lattice requires the doubling of lattice sites for both bosons and fermions, with the lattice spacing halved from $a$ to $a / 2$, the alternating sign structure for the fermion fields, and, to preserve hermiticity, a relative shift of $a / 4$ of the boson and fermion lattice sites so that ultimately the effective lattice spacing is $a / 4$. The price we had to pay for introducing supersymmetry is the doubling of both boson and fermion degrees of freedom. How to reduce them to the original number without spoiling supersymmetry is the next task. For this purpose we shall move from coordinate to momentum representation.

\section{Momentum Space}

Let us consider first the Fourier transform of the component fields $\psi(x)$ and $\varphi(x)$, and denote them by $\tilde{\psi}(p)$ and $\tilde{\varphi}(p)$ respectively. The lattice spacing being $a / 2$, the Brillouin zone extends 
over a $\frac{4 \pi}{a}$ interval and besides the physical states at $p=0$ will include doublers at $p=\frac{2 \pi}{a}$. Hence we have:

$$
\tilde{\varphi}\left(p+\frac{4 \pi}{a}\right)=\tilde{\varphi}(p), \quad \tilde{\psi}\left(p+\frac{4 \pi}{a}\right)=-\tilde{\psi}(p),
$$

where the minus sign in the case of $\tilde{\psi}$ is due to the $a / 4$ shift in coordinate space. The supersymmetry transformations (2.18) and (2.19) are then given by:

$$
\begin{aligned}
& \delta \tilde{\varphi}(p)=i \cos \frac{a p}{4} \alpha \tilde{\psi}(p), \\
& \delta \tilde{\psi}(p)=-i \frac{4}{a} \sin \frac{a p}{4} \alpha \tilde{\varphi}(p) .
\end{aligned}
$$

Eqs. (3.2) and (3.3) are consistent with both the periodicity conditions (3.1) and with the reality conditions expressed in momentum space by: $\tilde{\varphi}(p)^{\dagger}=\tilde{\varphi}(-p)$ and $\tilde{\psi}(p)^{\dagger}=\tilde{\psi}(-p)$.

Let us consider now the Fourier transform $\tilde{\Phi}(p)$ of the superfield $\Phi(x)$ given in (2.16). At each value of $p, \tilde{\Phi}(p)$ has a bosonic component $\tilde{\Phi}_{b}(p)$ and a fermionic one $\tilde{\Phi}_{f}(p)$ given by:

$$
\tilde{\Phi}_{b}(p)=\tilde{\varphi}(p), \quad \tilde{\Phi}_{f}(p)=\frac{a^{1 / 2}}{2} \tilde{\psi}\left(p+\frac{2 \pi}{a}\right) .
$$

The periodicity in $p$ of $\tilde{\Phi}_{b}(p)$ and $\tilde{\Phi}_{f}(p)$ are the same as $\tilde{\varphi}(p)$ and $\tilde{\psi}(p)$ respectively. The supersymmetry transformations can be easily written in terms of $\tilde{\Phi}(p)$ :

$$
\delta \tilde{\Phi}(p)=-2 i \alpha a^{-1 / 2} \cos \frac{a p}{4} \tilde{\Phi}\left(p+\frac{2 \pi}{a}\right)
$$

which is equivalent to (3.2) and (3.3). The physical fields are fluctuations around $p=0$ of the bosonic component $\tilde{\Phi}_{b}(p)$, and around $p=-\frac{2 \pi}{a}$ for the fermionic component $\tilde{\Phi}_{f}(p)$. In terms of $\tilde{\Phi}$, these two physical degrees of freedom have a natural interpretation of being species doublers to each other. The configurations at $p=0$ and $p=-\frac{2 \pi}{a}$ correspond respectively to constant and alternating sign configurations on the lattice of spacing $\frac{a}{2}$, as discussed above. Large fluctuations are, however, allowed on the lattice with the result of doubling the number of degrees of freedom with respect to the original lattice of spacing $a$. In particular doublers at $p=0$ and $p=-\frac{2 \pi}{a}$ with the "wrong" statistics will appear. In order to reduce the degrees of freedom to the original number it appears most natural at this point to introduce a cutoff on the momentum, limiting the bosonic modes to the standard Brillouin zone $\left(-\frac{\pi}{a}, \frac{\pi}{a}\right)$ and the fermionic ones to $\left(-\frac{3 \pi}{a},-\frac{\pi}{a}\right)$. In other words wavelengths shorter that $a$ will correspond to fermionic degrees of freedom, wavelengths longer than $a$ to bosonic degrees of freedom. This amounts to impose the constraints

$$
\tilde{\Phi}_{b}(p)=0 \quad p \in\left\{-\frac{3 \pi}{a},-\frac{\pi}{a}\right\} \quad \text { and } \quad \tilde{\Phi}_{f}(p)=0 \quad p \in\left\{-\frac{\pi}{a}, \frac{\pi}{a}\right\}
$$

These constraints are local in momentum space, hence highly non local in coordinate space and they allow to express the value of the fields in the half-integer multiples of $a$ in terms of the values in the integer multiples. For example, from the first of (3.6):

$$
\varphi\left(m a+\frac{a}{2}\right)=\frac{1}{2 \pi} \sum_{n} \frac{(-1)^{n-m}}{m-n+\frac{1}{2}} \varphi(n a) .
$$

Non locality in this case does not arise, as in the SLAC derivative, from the definition of the derivative on the lattice, but rather from the definition of the supersymmetric covariant derivative, which involve finite differences over a $\frac{a}{2}$ spacing. 


\section{The Action}

As remarked at the beginning the only possible superfield action for this system is the free one given in (2.4). This can be put on the lattice in superfield notation as:

$$
S=\frac{a}{2 \pi} \int_{-\frac{3 \pi}{a}}^{\frac{\pi}{a}} d p \tilde{\Phi}(-p) \sin \frac{a p}{4} \sin \frac{a p}{2} \tilde{\Phi}(p) .
$$

The different factors in (4.1) are in one to one correspondence with the terms in (2.4): $\sin \frac{a p}{4}$ is a finite difference of spacing $\frac{a}{2}$ and corresponds to the super derivative $D$ while $\sin \frac{a p}{2}$ is the lattice version of the normal derivative $\frac{\partial}{\partial x}$. The superfield $\tilde{\Phi}(p)$ is the one given in (3.4) and the constraints (3.6) are understood. For each value of $p, \tilde{\Phi}(p)$ is purely bosonic or fermionic. The integration region covers the bosonic range $\left\{-\frac{\pi}{a}, \frac{\pi}{a}\right\}$ and the fermionic range $\left\{-\frac{3 \pi}{a},-\frac{\pi}{a}\right\}$. The action changes sign when $p \rightarrow p+\frac{4 \pi}{a}$, so an integration over the whole $\frac{8 \pi}{a}$ would identically vanish. It can be checked directly that the action 4.1) is invariant under the supersymmetry transformations (3.5). In terms of component fields (4.1) can be written as:

$$
S=\frac{a}{2 \pi} \int_{-\frac{\pi}{a}}^{\frac{\pi}{a}} d p\left[\tilde{\varphi}(-p) \sin \frac{a p}{4} \sin \frac{a p}{2} \tilde{\varphi}(p)-\frac{a}{4} \tilde{\psi}(-p) \cos \frac{a p}{4} \sin \frac{a p}{2} \tilde{\psi}(p)\right]
$$

and it is invariant under SUSY transformations (3.2) and (3.3). The fields in (4.2) are defined in the $\left\{-\frac{\pi}{a}, \frac{\pi}{a}\right\}$ range of the momentum and can be associated in the coordinate space to fields defined on a lattice of spacing $a^{3}$. Let us denote them $\hat{\varphi}(a n)$ and $\hat{\psi}(a m)$ to distinguish them from $\varphi(x)$ and $\psi(x)$ defined on a lattice of spacing $\frac{a}{2}$. A simple Fourier transform allows then to write the action (4.2) in the coordinate space, exhibiting its non local nature:

$$
\begin{aligned}
S=\sum_{n, m} \frac{2 \sqrt{2}}{2 \pi}[ & \hat{\varphi}(\text { am }) \hat{\varphi}(\text { an })(-1)^{m-n}\left(-\frac{3}{16(m-n)^{2}-9}+\frac{1}{16(m-n)^{2}-1}\right) \\
& \left.-i \hat{\psi}(\text { am }) \hat{\psi}(\text { an })(-)^{m-n} a(m-n)\left(\frac{1}{16(m-n)^{2}-9}+\frac{1}{16(m-n)^{2}-1}\right)\right] .
\end{aligned}
$$

\section{Some Conclusions}

This simple one dimensional model suggests that, within the extended lattice of the link approach, component fields of a superfield expansion are associated to different regions of the Brillouin zone as if they were species doublers of each other. Momentum representation has a privileged role in this approach ${ }^{4}$, and an exact supersymmetric action can easily be constructed in this representation. The price to be paid is some non locality in the definition of the supersymmetric transformations and ultimately of the action when the coordinate representation is used. This model

\footnotetext{
${ }^{3}$ Notice however that if the momentum integration in (4.2) was extended to the whole Brillouin zone of the extended lattice, that is the interval $\left\{-\frac{3 \pi}{a}, \frac{\pi}{a}\right\}$, the result would be identical to the one given in (4.2). In fact the doublers at $p=\frac{2 \pi}{a}$ have the "wrong" statistics with respect to the symmetry of the lagrangian density, and the integral over the interval $\left\{-\frac{3 \pi}{a},-\frac{\pi}{a}\right\}$ vanishes identically. The constraints (3.6) may then be regarded as superfluous: bosonic modes with wavelength less than $a$ and fermionic modes with wavelength longer than $a$ naturally decouple.

${ }^{4}$ Momentum representation has been used in studying supersymmetric theories in low dimension. See for instance [10] and, for some recent developments $[11,12]$.
} 
is very simple, in the sense that it contains only one supersymmetric charge with no interaction. So the extension to higher dimensions, or at least to extended supersymmetries in one dimension is essential.

This will probably require a non commutative lattice. the argument is the following: consider a $D=2, N=1$ supersymmetry with supersymmetry algebra $Q_{1}^{2}=\frac{\partial}{\partial x}, Q_{2}^{2}=\frac{\partial}{\partial y}$ and $\left\{Q_{1}, Q_{2}\right\}=0$. Then a superfield expansion on the lattice in the spirit of (2.10) would be:

$$
\Phi(x, y)=\varphi(x, y)+(-1)^{\frac{2 x}{a}} \psi_{1}(x, y)+(-1)^{\frac{2 y}{a}} \psi_{2}(x, y)+(-1)^{\frac{2 x}{a}}(-1)^{\frac{2 y}{a}} F(x, y) .
$$

For this superfield to generate a consistent supersymmetric interaction term the sign factors $(-1)^{\frac{2 x}{a}}$ and $(-1)^{\frac{2 y}{a}}$ have to anticommute (just as $\theta_{1}$ and $\theta_{2}$ would). This would imply non-commutative space-time on the lattice, giving

$$
[x, y]=\frac{i}{4 \pi} a^{2}
$$

with commutativity recovered in the continuum limit $a \rightarrow 0$. The relevance of non commutative lattices in the link approach has already been considered in [13].

\section{Acknowledgments}

This work is supported in part by Japanese Ministry of Education, Science, Sports and Culture under the grant No. 18540245 and INFN research fund. I.K. is supported by the Nishina Memorial Foundation.

\section{References}

[1] S. Catterall, D. B. Kaplan and M. Unsal, arXiv:0903.4881 [hep-lat].

[2] A. D’Adda, I. Kanamori, N. Kawamoto and K. Nagata, Nucl. Phys. B 707, 100 (2005) [arXiv:hep-lat/0406029].

[3] A. D'Adda, I. Kanamori, N. Kawamoto and K. Nagata, Phys. Lett. B 633, 645 (2006) [arXiv:hep-lat/0507029].

[4] A. D’Adda, I. Kanamori, N. Kawamoto and K. Nagata, Nucl. Phys. B 798, 168 (2008) [arXiv:0707.3533 [hep-lat]].

[5] S. Arianos, A. D'Adda, A. Feo, N. Kawamoto and J. Saito, arXiv:0806.0686 [hep-lat], to appear on IJMPA.

[6] P. H. Damgaard and S. Matsuura, JHEP 0709, 097 (2007) [arXiv:0708.4129 [hep-lat]].

[7] F. Bruckmann and M. de Kok, Phys. Rev. D 73 (2006) 074511 [arXiv:hep-lat/0603003].

[8] F. Bruckmann, S. Catterall and M. de Kok, Phys. Rev. D 75 (2007) 045016 [arXiv:hep-lat/0611001].

[9] A. D’Adda, N. Kawamoto and J. Saito, arXiv:0907.4137 [hep-th].

[10] M. Hanada, J. Nishimura and S. Takeuchi, Phys. Rev. Lett. 99 (2007) 161602 [arXiv:0706.1647 [hep-lat]].

[11] D. Kadoh and H. Suzuki, arXiv:0909.3686 [hep-th].

[12] G. Bergner, arXiv:0909.4791 [hep-lat].

[13] S. Arianos, A. D’Adda, N. Kawamoto and J. Saito, PoS LAT2007, 259 (2007) [arXiv:0710.0487 [hep-lat]]. 